\title{
Auxílio da tomografia computadorizada e da rinoscopia no diagnóstico das afecções nasais de cães: revisão
}

\author{
Computerized axial tomography and rhinoscopy as \\ diagnostic aids in canine nasal disorders: a review
}

Fernanda de Assis Bueno Auler ${ }^{[a]}$, Leda Marques de Oliveira-Barros ${ }^{[a]}$,Ana Carolina Brandão de Campos Fonseca Pinto ${ }^{[b]}$, Angelo João Stopiglia [c]

[a] Pós-graduandas do Departamento de Cirurgia da Faculdade de Medicina Veterinária e Zootecnia da Universidade de São Paulo, São Paulo, SP - Brasil, e-mail: feauler@usp.br; fe_bueno@hotmail.com; ledamobarros@usp.br

[b] Professora Doutora do Departamento de Cirurgia da Faculdade de Medicina Veterinária e Zootecnia da Universidade de São Paulo, São Paulo, SP - Brasil, e-mail: anacarol@usp.br

[c] Professor titular do Departamento de Cirurgia da Faculdade de Medicina Veterinária e Zootecnia da Universidade de São Paulo, São Paulo, SP - Brasil, e-mail: stopa@usp.br

\section{Resumo}

As afecções nasais em cães possuem causas variadas e algumas vezes cursam com sinusites, sendo raros os casos em que não há doença nasal pré-existente. 0 diagnóstico presuntivo é baseado em manifestações clínicas. Entre as afecções nasais caninas mais comuns estão a rinite inflamatória, a rinite bacteriana, a rinite fúngica, a neoplasia, o corpo estranho, o trauma, a rinite parasitária e a rinite secundária às afecções dentárias. A tomografia computadorizada e a rinoscopia, quando associadas, auxiliam no diagnóstico, na melhor compreensão da doença e no favorecimento da escolha do tratamento. Esta revisão tem como objetivo contribuir para o conhecimento da utilização da tomografia e da rinoscopia no diagnóstico das afecções nasais em cães.

Palavras-chave: Tomografia computadorizada. Rinoscopia. Afecções nasais. Cavidade nasal. Cães. Rinite Canina.

\section{Abstract}

Canine nasal disorders have various causes, at times coexist with sinusitis and rare are the cases in which there is no preexisting nasal disease. Presumptive diagnosis is based on clinical manifestations. Inflammatory rhinitis, bacterial rhinitis, fungal rhinitis, neoplasm, foreign body, traumatism, parasitic rhinitis and secondary rhinitis to dental conditions are among the most common nasal disorders. Computerized axial tomography associated with rhinoscopy contributes to diagnosis, to a better understanding of the disease and to the choice of treatment. This review aims to assess the contribution of computerized axial tomography and rhinoscopy to the diagnosis of canine nasal disorders.

Keywords: Computerized tomography. Rhinoscopy. Nasal disorders. Nasal cavity. Dogs. Canine rhinitis. 


\section{Introdução}

As afecções nasais em cães possuem causas variadas e são classificadas como rinites primárias quando há causas alérgicas, bacterianas, fúngicas e neoplásicas, ou secundárias em casos de traumatismos, corpos estranhos, parasitas e afecções dentárias (DAVIDSON et al., 2004; FORRESTER et al., 2002; McCARTHY; McDEIRMAN, 1990). 0 diagnóstico presuntivo é baseado nas manifestações clínicas, como secreção nasal uni ou bilateral, esternutações, esternutações reversas paroxísticas, engasgos, halitose, epistaxes, tosse, distrição respiratória, dor e deformação facial (McCARTHY; McDEIRMAN, 1990).

Exames de imagem da cavidade nasal e dos seios paranasais são úteis como componentes na avaliação de pacientes com indícios de doença nasal, sendo o objetivo permitir visibilizar a extensão da lesão e avaliar o acometimento das estruturas (DAVIDSON et al., 2004; LEFEBVRE et al., 2005; SAUNDERS et al., 2004). De acordo com a literatura sobre afecções nasais, mesmo com exames de imagem de grande acurácia e especificidade, recomenda-se a utilização da rinoscopia, que pode confirmar a existência de afecção previamente visibilizada pelos exames de imagem radiográficos, tomográficos e/ou ressonância magnética (DAVIDSON et al., 2004; McCARTHY, 2005; SAUNDERS et al., 2004; VENKER-VAN HAAGEN, 2005). As culturas e os exames citológicos e histopatológicos também devem ser utilizados para a conclusão diagnóstica (DAVIDSON et al., 2004; McCARTHY, 2005; SAUNDERS et al., 2004).

Esta revisão tem como objetivo contribuir para o conhecimento da utilização de exames da tomografia computadorizada e da rinoscopia como meios complementares no diagnóstico nas principais afecções nasais em cães.

\section{Aspectos gerais}

\section{Tomografia computadorizada e rinoscopia}

O simples exame radiográfico de cabeça é utilizado como o primeiro exame de imagem nos casos de suspeita de rinite, não permitindo, contudo, visibilizar com precisão a extensão e o envolvimento das estruturas relacionadas com a afecção presente, em virtude da sobreposição de imagens (DAVIDSON et al., 2004; LEFEBVRE et al., 2005).

A tomografia computadorizada de cabeça (TCC) apresenta vantagens em relação à radiografia convencional, pois permite, por meio de cortes transversais, avaliar detalhadamente a cavidade nasal e os seios paranasais (BURK, 1992; DOUST; SULLIVAN, 2004; KUEHN, 2006; PARK; BECK; LeCOUTEUR, 1992; SCHWARTZ, 1995), melhorando a capacidade como auxílio diagnóstico de doenças nasais de forma mais precisa (DAVIDSON et al., 2004; LEFEBVRE et al., 2005; WITHROW, 1989). Com o ajuste da escala de contraste, é possível incrementar a discriminação das estruturas de tecidos moles, daquelas que são apenas fracamente mineralizadas, facilitando a visibilidade das estruturas turbinadas na parte rostral da cavidade nasal (DAVIDSON et al., 2004). Com a TCC, é possível observar imagens que permitem avaliar a extensão da lesão e o envolvimento ou não das estruturas anatômicas, como a região das conchas nasais, dos maxiloturbinados e etmoturbinados, do septo nasal, do vômer, do osso nasal e maxilar, da nasofaringe, do meato nasofaríngeo, da lâmina cribiforme, dos seios frontais, do seio esfenoidal, do recesso maxilar, do palato, da região periorbital e arco zigomático, e do alvéolo dentário (JOHNSON; WISNER, 2007; LEFEBVRE et al., 2005; THRALL et al., 1989; WITHROW, 1989). Existe, ainda, a possibilidade de se administrar contraste por via intravenosa como recurso na delimitação das margens tumorais, auxiliando na diferenciação da existência de opacificação, melhorando a acurácia do exame tomográfico (JOHNSON et al., 2007). Porém, na Medicina Veterinária há limitações, como a necessidade de anestesia para a execução do exame, o custo do exame, o local específico para o equipamento, além da manutenção e do custo do equipamento (DAVIDSON et al., 2004; JOHNSON et al., 2007).

Tempos antes da utilização da rinoscopia, cirurgias exploratórias de grande extensão nas cavidades nasais e seios paranasais eram realizadas com o intuito de estabelecer o diagnóstico. Porém, elas apresentavam maior taxa de morbidade e/ou mortalidade, principalmente quando comparadas com a rinoscopia, considerado um exame seguro e minimamente invasivo (DAVIDSON et al., 2004; ELIE; SABO, 2006; FORBES LENT; HAWKINS, 1992; McCARTHY; McDEIRMAN, 1990). Comumente, exames laboratoriais e de imagem são realizados previamente à 
rinoscopia, que tem como intuito confirmar a existência de lesão observada a priori (McCARTHY, 2005). 0 equipamento de endoscopia pode ser de fibra óptica rígida ou flexível e a dimensão da cavidade nasal pode impor algumas limitações ao exame, sendo necessário utilizar mais de um tipo de equipamento endoscópico (FORD, 1990). Nos casos dos cães de médio e grande porte, a acessibilidade é maior. No entanto, independente do tamanho do paciente ou do tipo de equipamento, tanto flexível como rígido, a rinoscopia não permite a visibilidade de toda a extensão da cavidade nasal (McCARTHY, 2005).

Hemograma completo com contagem plaquetária, perfis de coagulação e possível transfusão sanguínea também devem ser considerados de acordo com a gravidade da doença, pois algum grau de hemorragia durante a rinoscopia pode ser inevitável (DAVIDSON et al., 2004; McCARTHY, 2005). A rinoscopia realizada com acesso rostral à cavidade nasal, também denominada rinoscopia anterior, permite a visibilidade e a inspeção de septo nasal, meato nasal comum dorsal, médio, ventral, conchas nasal dorsal, ventral e dos etmoturbinados.

Dependendo do tipo constitucional do paciente, é possível chegar à nasofaringe, porém na maioria dos casos, a inspeção da nasofaringe ocorre por meio da rinoscopia posterior ou nasofaringoscopia (McCARTHY, 2005), a qual permite a visibilidade direta da nasofaringe pela técnica de retroflexão do endoscópio flexível (DAVIDSON et al., 2004; WILLARD; RADLINSKY, 1999). Durante o exame, são avaliados a presença e o tipo de secreção, inflamação das conchas nasais, presença de pólipos, proliferações teciduais, corpos estranhos, placas fúngicas e alterações anatômicas significativas, levando-se em consideração a região acometida e o envolvimento uni ou bilateral (McCARTHY, 2005; NELSON; COUTO, 2001; VENKER-VAN HAAGEN, 2005; WILLARD, RADLINSKY, 1999).

Além disso, também é útil como forma terapêutica em casos de remoção de corpos estranhos. Sua contribuição se estende, ainda, na coleta de material para realização de exame citológico e estudo histopatológico, sob orientação visual, obtendo-se uma compreensão mais detalhada da natureza do processo da doença, aumentando a probabilidade de se concluir o diagnóstico (DAVIDSON et al., 2004; McCARTHY; McDEIRMAN, 1990; VENKERVAN HAAGEN, 2005). Apesar de ser um exame específico, efetivo e pouco invasivo, tem como desvantagem o fato de não trazer informações precisas quanto à extensão da lesão, independentemente do porte do paciente. 0 equipamento utilizado e a dimensão da cavidade nasal podem impor algumas limitações ao exame, sendo necessário utilizar mais de um tipo de equipamento endoscópico (FORD, 1990), além da obrigatoriedade de anestesia geral, esta também presente nos exames tomográficos. A visibilização da cavidade nasal pode ser prejudicada quando há presença de secreções mucopurulentas espessas ou sanguinolentas, o que ocorre na maioria dos casos. Por fim, outra desvantagem é o custo elevado na obtenção e manutenção dos equipamentos (DAVIDSON et al., 2004; McCARTHY, 2005).

\section{Principais afecções nasais}

0 trato respiratório nos cães é a maior porta de entrada para agentes infecciosos, e também torna possível a entrada de corpo estranho, o trauma e o desenvolvimento de neoplasias (FORD, 1990).

Em cães com rinite inflamatória, ou rinite linfoplasmocitária, a imagem tomográfica pode indicar opacificação, lise óssea de turbinados, septo nasal e vômer, e acometimento de seio frontal, conforme a cronicidade da doença (JOHNSON; WISNER, 2007; LEFEBVRE; et al., 2005; WINDSOR et al., 2004). Na rinoscopia, pode-se visibilizar presença de secreção mucoide ou mucopurulenta, hiperemia, edema, erosões, e friabilidade na mucosa acompanhada, em alguns casos, de atrofia ou ausência de conchas nasais (WINDSOR et al., 2004). Nos casos de rinite fúngica, principalmente quando causada pelo Aspergillus spp., a progressão da doença nos animais afetados inicia-se na cavidade nasal e, como regra, estende-se para os seios frontais, tornando-se afecção sinonasal decorrente da endotoxina produzida pelo fungo (BENITAH, 2006).

Essa afecção é considerada, por muitos autores, como relativamente comum nos cães (BENITAH, 2006; JOHNSON et al., 2006; VENKER-VAN HAAGEN, 2005). Nesses casos, é possível visualizar extensa destruição dos turbinados (Figura 1) e áreas hipoatenuantes nas passagens nasais, por meio do exame tomográfico (LEFEBVRE et al., 2005). A rinoscopia permite, em função da destruição de maxiloturbinados e etmoturbinados, o acesso ao seio frontal e, 
normalmente, a visibilidade de necrose tecidual e placas fúngicas na cavidade nasal ou no seio frontal (Figuras 1 e 2) durante o exame. 0 diagnóstico é concluído com a cultura das placas fúngicas retiradas durante a rinoscopia e a biópsia (BENITAH, 2006).

Por meio da tomografia computadorizada de cabeça, imagens de lise em conchas nasais, lise em septo nasal e vômer, acometimento de nasofaringe, palato, lise em placa cribriforme e de outras estruturas adjacentes, como osso maxilar e osso nasal, envolvimento de órbita (Figura 3) e ou do espaço retrobulbar sugerem presença de neoplasia. Entretanto, não se pode afirmar a existência de um único achado que caracterize definitivamente essa afecção (BURK, 1992).

Nesses casos, por meio da rinoscopia, é possível visibilizar a proliferação tecidual com superfície de coloração esbranquiçada, rósea ou vinhosa, diferente das estruturas normais da cavidade nasal (Figura 4), e seu aspecto pode variar: ser irregular,

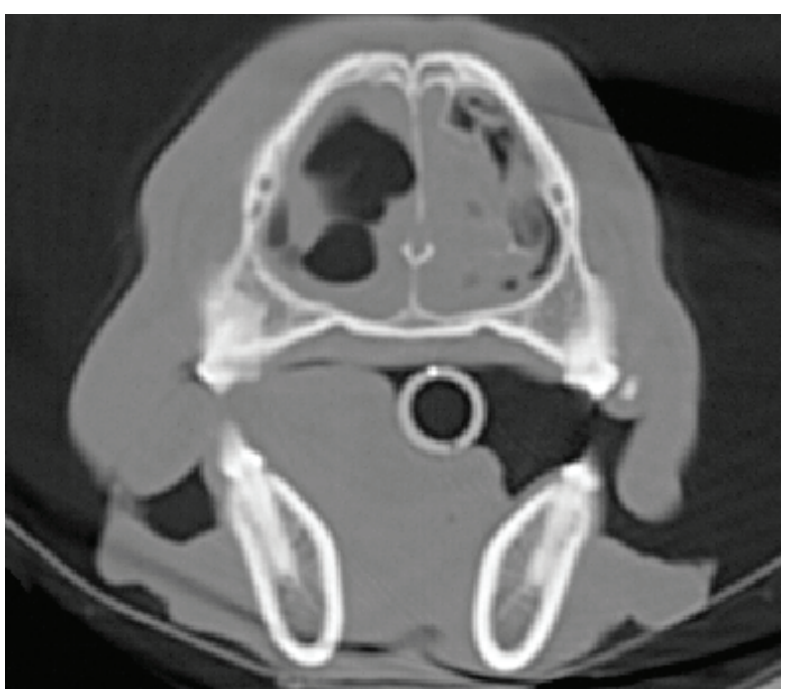

Figura 1 - Imagem tomográfica (corte transversal) da cabeça em nível de cavidades nasais e seio frontal de cão, SRD, macho, 5 anos, com diagnóstico conclusivo de aspergilose nasal: observa-se destruição de conchas nasais do lado direito, lise em vômer e septo nasal, opacificação da passagem nasal esquerda rostralmente. Para estes tomogramas, foram utilizadas seleções de janela e nível para avaliação de tecido ósseo.

Fonte: Serviço de Diagnóstico por Imagem do Hospital Veterinário da Faculdade de Medicina Veterinária e Zootecnia da Universidade de São Paulo,SP, 2010.

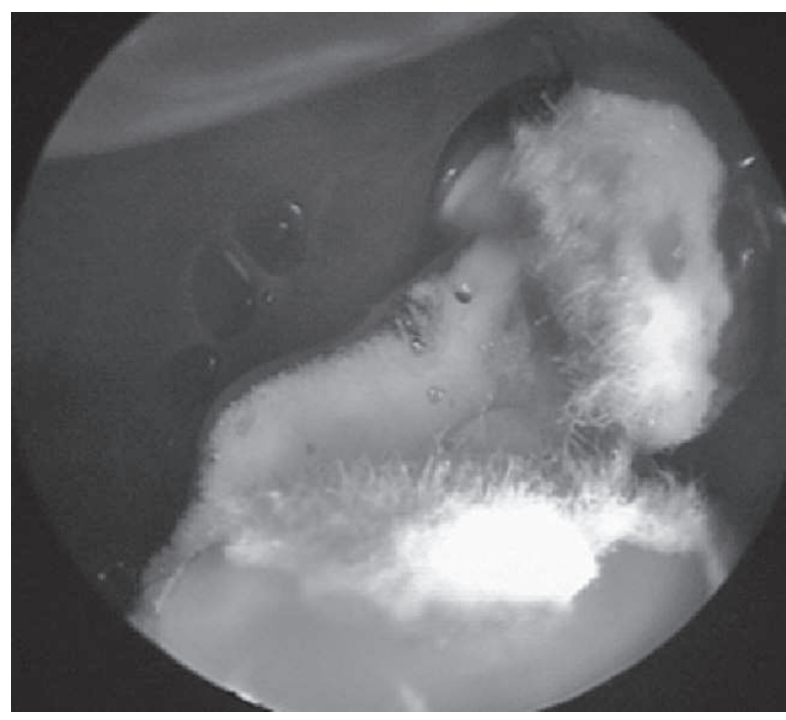

Figura 2 - Imagem durante a rinoscopia de cão, SRD, 10 anos, macho com diagnóstico de rinite aspergilose sionasal: observa-se placa fúngica na região de seio frontal.

Fonte: São Paulo - SP. YOSHITOSHI; AULER, 2007.

lisa, vascularizada ou não, e de aparência cística, sólida, ou polipoide. Observa-se também, em conjunto, fibrina ou necrose e inflamação ao redor da neoplasia, o que pode dificultar o resultado da coleta pela rinoscopia (McCARTHY, 2005). Em virtude da grande variabilidade no aspecto das neoplasias nasais, torna-se necessário o estudo histopatológico do material coletado a fim de determinar o tipo de tumor (McCARTHY, 2005). Carcinomas, adenocarcinomas e carcinoma de células escamosas compreendem cerca de dois terços dos tipos de neoplasia encontrados nesses casos (LANA; WITHROW, 2001). Tumores venéreos transmissíveis e neoplasias de origem mesenquimal (osteocondroma, fibrossarcoma e osteossarcoma) também acometem a cavidade nasal (DAVIDSON et al., 2004).

0 exame de imagem de corpos estranhos nasais nem sempre permite visibilizar o objeto, podendo haver confusão na interpretação diagnóstica (McCARTHY, 2005; VENKER-VAN HAAGEN, 2005). A tomografia computadorizada de cabeça pode apresentar imagens diretas, sugerindo a presença do corpo estranho e a localização no interior da cavidade nasal, ou imagens de lise óssea no local do corpo estranho alojado, principalmente em casos crônicos (JOHNSON et al., 2007). Durante a rinoscopia, 


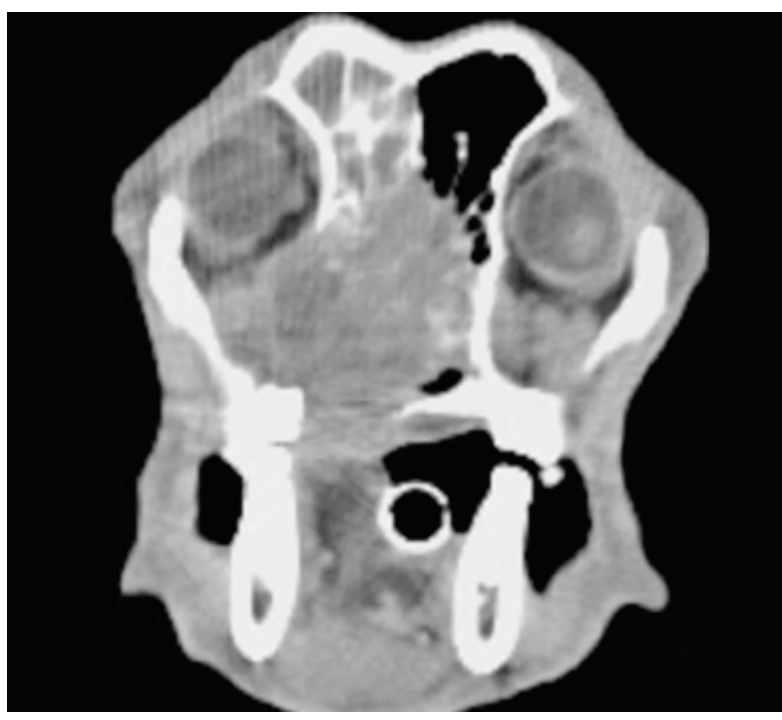

Figura 3 - Imagem tomográfica (corte transversal) da cabeça (na região das órbitas) de um cão, Golden Retriever, macho, 10 anos, com diagnóstico conclusivo de carcinoma. Presença de massa de aspecto heterogêneo com áreas hipoatenuantes que promove lise dos etmoturbinados, placa cribiforme e vômer, palato duro, parede óssea da órbita, osso frontal e pterigoide. A massa invade órbita do lado direito, deslocando dorso-lateralmente o olho. Observa-se também o comprometimento do seio frontal direito, que se apresenta preenchido por material com atenuação de partes moles. Para estes tomogramas, foram utilizadas seleções de janela e nível para avaliação de tecidos moles.

Fonte: Serviço de Diagnóstico por Imagem do Hospital Veterinário da Faculdade de Medicina Veterinária e Zootecnia da Universidade de São Paulo, SP, 2010.

a presença de grande quantidade de secreção causada pela presença do corpo estranho pode dificultar a visibilidade. Pequenos corpos estranhos na cavidade nasal podem ser removidos rostralmente, com pinça própria para remoção destes ou fórceps de biópsia. Já nos casos de corpos estranhos de dimensão maior, é indicado que estes sejam empurrados para a nasofaringe para serem removidos. A cirurgia pode ser executada nos casos de insucesso (McCARTHY, 2005).

A doença dental pode causar rinite uni ou bilateral. A tomografia computadorizada usualmente apresenta imagens do canino superior ou mais dentes acometidos e osteólise periapical. Além disso,

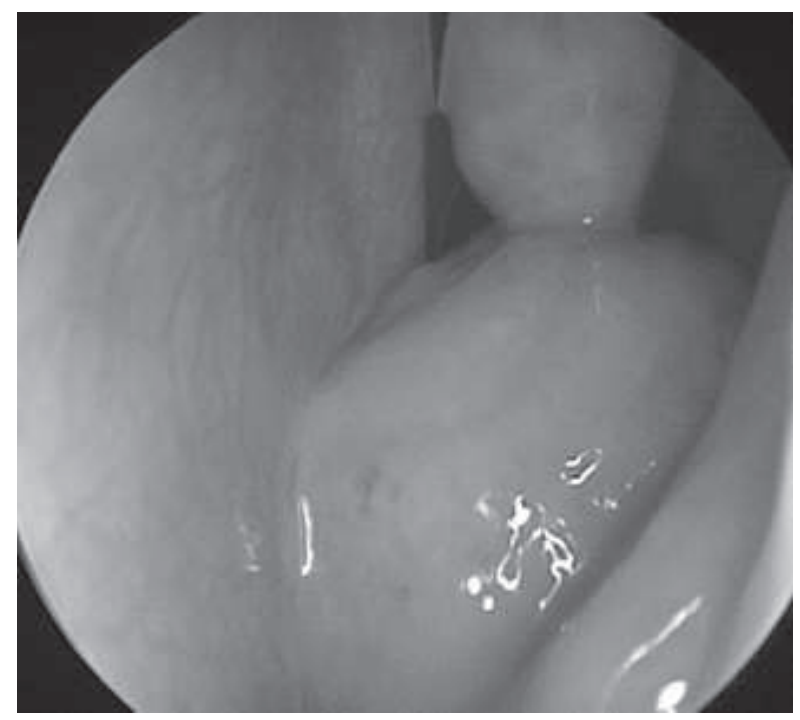

Figura 4 - Imagem da rinoscopia de cão, Pastor Belga, 8 anos, macho com diagnóstico conclusivo de adenocarcinoma: observa-se proliferação tecidual rósea clara, em terço posterior médio ventral e etmoturbinados em cavidade nasal esquerda, septo nasal.

Fonte: São Paulo - SP. Fernanda Auler, 2008.

ainda podem ser visibilizadas áreas de opacificação, lise óssea em palato e turbinados que podem confundir-se com imagens sugestivas de neoplasia (KUEHN, 2006). Na rinoscopia, são observadas secreções mucopurulentas ou sanguinolentas, presença de tecido com aspecto de fibrina, edema de conchas nasais, algumas vezes proliferações teciduais localizadas aproximadamente da raiz do dente afetado e a diminuição das passagens aéreas (VENKER-VAN HAAGEN, 2005). A incidência de rinite secundária à doença dental vem decrescendo com o aumento dos cuidados, higiene e tratamento bucal periódico que vêm ocorrendo nos pequenos animais (McCARTHY, 2005).

\section{Considerações finais}

Estudos realizados sobre a utilização da tomografia computadorizada de cabeça e a rinoscopia nos casos de afecções nasais contribuem para o diagnóstico. A tomografia computadorizada permite visibilizar aspectos que nem sempre podem ser detectados pela rinoscopia como detalhamento da extensão da lesão e das estruturas acometidas, além de auxiliar na 
identificação de áreas afetadas, não se podendo indicar, porém, a existência de um único achado observado pelo exame tomográfico que caracterize definitivamente o tipo de afecção, já que imagens como opacificação, lise óssea, entre outras, também podem estar presentes em diferentes tipos de rinites.

A rinoscopia vem sendo utilizada com maior ênfase na Medicina Veterinária, na atualidade, e sua grande vantagem é a visibilidade direta das cavidades nasais, permitindo, na maioria dos casos, confirmar o tipo de afecção sugerida pelos exames de imagem. Além disso, é útil como forma terapêutica e na coleta de material, auxiliando, como consequência, no diagnóstico e no tratamento.

\section{Agradecimentos}

O presente trabalho foi realizado com o apoio do Conselho Nacional de Desenvolvimento Científico e Tecnológico - CNPq - Brasil.

\section{Referências}

BENITAH, N. Canine nasal Aspergillosis. Clinical Techniques in Small Animal Practice, v. 21, n. 2, p. 82-88, 2006.

BURK, R. L. Computed tomographic imaging of nasal disease in 100 dogs. Veterinary Radiology Ultrassonography, v. 33, p. 177-180, 1992.

DAVIDSON, A. P. et al. Doenças do nariz e dos seios nasais. In: ETTINGER, S. J.; FELDMAN, E. C. Tratado de medicina interna veterinária: doenças do cão e do gato. 5. ed. Rio de Janeiro: Guanabara Koogan, 2004. p. 1059-1082.

DOUST, R.; SULLIVAN, M. Nasal discharge, sneezing, and reverse sneezing. In: KING, L. G. Textbook of respiratory diseases in dogs and cats. Saint Louis: Elsevier Saunders, 2004. p. 17-28.

ELIE, M.; SABO, M. Basics in canine and feline rhinoscopy. Clinical Techniques in Small Animal Practice, v. 21, n. 2, p. $60-63,2006$.

FORBES LENT, S. E.; HAWKINS, E. C. Evaluation of rhinoscopy and rhinoscopy-assisted mucosal biopsy in diagnosis of nasal disease in dogs: 119 cases (1985-1989). Journal of American Veterinary Medical Association, v. 201, p. 1425-1429, 1992.
FORD, R. B. Endoscopy of the upper respiratory tract of the dog and cat. In: TAMS, T. R. Small animal endoscopy. Saint Louis: Mosby Company, 1990.

FORRESTER, S. D.; JONES, J. C. Identifying the cause of nasal diseases in dogs. Veterinary Medicine, v. 97, n. 7, p. $530-542,2002$.

JOHNSON, L. R. et al. Results of rhinoscopy alone or in conjunction with sinuscopy with aspergilosis: 46 cases (20012004). Journal of American Veterinary Association, v. 228, n. 5, p. 738-742, 2006.

JOHNSON, E. G.; WISNER, E. R. Advances in respiratory imaging. Veterinary Clinics of North America: Small Animal Practice, v. 37, n. 5, p. 879-900, 2007.

KUEHN, N. F. Nasal computed tomography. Clinical Techniques in Small Animal Practice, v. 21, n. 2, p. 5559, 2006.

LANA, S. E.; WITHROW, S. J. Tumors of the respiratory system - nasal tumors. In: WITHROW, S. J.; Mac EWEN, E. G. (Ed.). Small animal clinical oncology. 3rd ed. Philadelphia: Saunders, 2001. p. 370-377.

LEFEBVRE, J. et al. Computed tomography as an aid in the diagnosis of chronic nasal disease in dog. Journal of Small Animal Practice, v. 46, n. 6, p. 280-285, 2005.

McCARTHY, T. C.; McDEIRMAN, S. L. Rhinoscopy. Veterinary Clinics of North America: Small Animal Practice, v. 20, n. 5, p. 1265-1290, 1990.

McCARTHY, T. C. Veterinary endoscopy for the small animal practitioner. Saint Louis: Elsevier Saunders, 2005.

NELSON, R. W.; COUTO, C. G. Medicina interna de pequenos animais. 2. ed. Rio de Janeiro: Guanabara Koogan, 2001.

PARK, R. D. et al. Comparison of computed tomography and radiography for detecting changes induced by malignant nasal neoplasia in dogs. Journal of American Veterinary Medical Association, v. 201, n. 11, p. 17201724, 1992.

SAUNDERS, J. et al. Radiographic, magnetic resonance imaging, computed tomographic, and rhinoscopic features of nasal aspergillosis in dogs. Jounal of American Veterinary Association, v. 225, n. 11, p. 1703-1712, 2004.

SCHWARTZ, T. Comparison of sensitivity and specificity of conventional radiography and computed tomographic in nasal tumors and fungal rhinitis in dogs. Veterinary Radiology \& Ultrasound, v. 36, p. 428, 1995. 
THRALL, D. E. et al. A comparison of radiographic and computed tomographic findings in 31 dogs with malignant nasal cavity tumors. Veterinary Radiology, v. 30, n. 2, p. 59-66, 1989.

VENKER-VAN HAAGEN, A. J. The nose and nasal sinuses. In: VENKER-VAN HAAGEN, A. Ear, nose throat, and tracheobronchial diseases in dogs and cats. Hannover: Schlütersche, 2005. p. 51-81.

WILLARD, M. D.; RADLINSKY, M. A. Endoscopy examination of choanae in dogs and cats: 118 cases. Journal of American Veterinary Association, v. 215, n. 9, p. 13011305, 1999.
WINDSOR, R. C. et al. Idiopathic linfoplasmocytic rhinits in 37 dogs: 1997-2002. Journal of American Veterinary Medical Association, v. 224, n. 12, p. 1952-1957, 2004.

WITHROW, S. J. Tumors of the respiratory system. In: WITHROW, S. J.; Mac EWEN, G. Clinical veterinary oncology. Philadelphia: Lippincott, 1989. p. 215- 233.

Recebido: 02/03/2011 Received: 03/02/2011

Aprovado: 28/09/2011 Approved: 09/28/2011 